\title{
Competing Ground States of the New Class of Halogen-Bridged Metal Complexes
}

\author{
Shoji Yamamoto \\ Department of Physics, Okayama University, Tsushima, Okayama 700-8530, Japan
}

(Received 7 October 1999)

\begin{abstract}
Based on a symmetry argument, we study the ground-state properties of halogen-bridged binuclear metal chain complexes. We systematically derive commensurate density-wave solutions from a relevant two-band Peierls-Hubbard model and numerically draw the the ground-state phase diagram as a function of electron-electron correlations, electron-phonon interactions, and doping concentration within the Hartree-Fock approximation. The competition between two types of charge-densitywave states, which has recently been reported experimentally, is indeed demonstrated.
\end{abstract}

PACS numbers: 71.10.Hf, 71.45Lr, 75.30.Fv, 03.65.Fd

Halogen-bridged transition-metal linear-chain complexes (MX chains; $\mathrm{M}=$ metal, $\mathrm{X}=$ halogen) have been a central subject in material science due to their low dimensionality and competing electron-electron (elel) and electron-phonon (el-ph) interactions, which lead to the variety of electronic states. PtX compounds [1] provided various topics of both chemical and physical interest, such as intense dichroism and unusual Raman spectra which originate in their mixed-valence ground states. NiX compounds [2 [4] with mono-valence magnetic ground states visualized the competition between the Peierls and Mott insulators. Nasu [5] gave a theoretical description of the ground-state competition. The Los Alamos group [6] extensively investigated potential ground states, including incommensurate density waves 17] and spin-frustrated novel antiferromagnetism [8].

In recent years, the new class of these family compounds (MMX chains), which consists of binuclear metal units bridged by halogen ions, has been stimulating further interest in this system. The presence of the metal- metal bonds allows us to expect a wider variety of electronic structures [9 11]. $\mathrm{K}_{4}\left[\mathrm{Pt}_{2}(\mathrm{pop})_{4} \mathrm{X}\right] \cdot n \mathrm{H}_{2} \mathrm{O}(\mathrm{X}=$ $\mathrm{Cl}, \mathrm{Br}, \mathrm{I} ;$ pop $\left.=\mathrm{P}_{2} \mathrm{O}_{5} \mathrm{H}_{2}\right)$ 12, 13 and $\mathrm{M}_{2}(\mathrm{dta})_{4} \mathrm{I}(\mathrm{M}=$ $\mathrm{Pt}$, Ni; dta $=\mathrm{CH}_{3} \mathrm{CS}_{2}$ ] [14 are both typical of the MMX compounds but their electronic properties look rather different. $\mathrm{Pt}_{2}(\mathrm{dta})_{4} \mathrm{I}$ shows metallic conduction above room temperature 11], whereas no metallic behavior has ever been found in the pop series. Their ground states are both charge density waves 90 11 but show different valence structures. The variety of ground states must be one of the most interesting consequences of intrinsic multi-band effects and competing el-el and el-ph interactions. Here, based on a symmetry argument [15, 17], we reveal rich phase diagrams of the one-dimensional (1D) two-band three-orbital Peierls-Hubbard model. The competition between the two distinct charge-density-wave states is indeed demonstrated. Antiferromagnetic ground states for the Ni complexes and the onset of further novel phases under hole doping are also predicted.

We introduce the 1D model Hamiltonian as

$$
\begin{aligned}
\mathcal{H} & =\sum_{n, s}\left[\left(\varepsilon_{\mathrm{M}}-\beta \delta_{1: n}\right) n_{1: n, s}+\left(\varepsilon_{\mathrm{M}}+\beta \delta_{2: n}\right) n_{2: n, s}+\varepsilon_{\mathrm{X}} n_{3: n, s}\right]+\frac{K}{2} \sum_{n}\left(\delta_{1: n}^{2}+\delta_{2: n}^{2}\right) \\
& -\sum_{n, s}\left[\left(t_{\mathrm{MX}}-\alpha \delta_{1: n}\right) a_{1: n, s}^{\dagger} a_{3: n, s}+\left(t_{\mathrm{MX}}+\alpha \delta_{2: n}\right) a_{2: n, s}^{\dagger} a_{3: n, s}+t_{\mathrm{MM}} a_{1: n, s}^{\dagger} a_{2: n-1, s}+\text { H.c. }\right] \\
& +\sum_{n}\left(U_{\mathrm{M}} n_{1: n,+} n_{1: n,-}+U_{\mathrm{M}} n_{2: n,+} n_{2: n,-}+U_{\mathrm{X}} n_{3: n,+} n_{3: n,-}\right) \\
& +\sum_{n, s, s^{\prime}}\left(V_{\mathrm{MX}} n_{1: n, s} n_{3: n, s^{\prime}}+V_{\mathrm{MX}} n_{2: n, s} n_{3: n, s^{\prime}}+V_{\mathrm{MM}} n_{1: n, s} n_{2: n-1, s^{\prime}}\right)
\end{aligned}
$$

where $n_{i: n, s}=a_{i: n, s}^{\dagger} a_{i: n, s}$ with $a_{i: n, s}^{\dagger}$ being the creation operator of an electron with spin $s= \pm$ (up and down) for the $\mathrm{M} d_{z^{2}}(i=1,2)$ or $\mathrm{Xp}_{z}(i=3)$ orbital in the $n$th MXM unit, respectively, and $\delta_{i: n}=u_{3: n}-u_{i: n}$ with $u_{i: n}$ being the chain-direction displacement of the metal $(i=1,2)$ or halogen $(i=3)$ ion in the $n$th MXM unit. $\alpha$ and $\beta$ are, respectively, the intersite and intrasite el-ph coupling constants, while $K$ is the metalhalogen spring constant. We assume, based on experimental observations, that the MM units are not deformed $\left(u_{1: n}=u_{3: n-1}\right) . \varepsilon_{\mathrm{M}}$ and $\varepsilon_{\mathrm{X}}$ are the on-site energies (elec- tron affinities) of isolated metal and halogen atoms, respectively. The electron hoppings between these levels are modeled by $t_{\mathrm{MM}}$ and $t_{\mathrm{MX}}$, whereas the el-el Coulomb interactions by $U_{\mathrm{M}}, U_{\mathrm{X}}, V_{\mathrm{MM}}$, and $V_{\mathrm{MX}}$. The symmetry group of the system is given by $\mathbf{G}=\mathbf{P} \times \mathbf{S} \times \mathbf{T}$, where $\mathbf{P}=\mathbf{L} \wedge \mathbf{C}_{2}$ is the space group of a linear chain with $\mathbf{L}$ being the $1 \mathrm{D}$ translation group whose basis vector is the unit-cell translation, $\mathbf{S}$ the group of spin-rotation, and $\mathbf{T}$ the group of time reversal. Let $G$ denote the irreducible real representations of $\mathbf{G}$, where their representation space is spanned by the Hermitian operators 
$\left\{a_{i: k, s}^{\dagger} a_{j: k^{\prime}, s^{\prime}}\right\}$. There is a one-to-one correspondence between $\check{G}$ and broken-symmetry phases of density-wave type. Any representation $\breve{G}$ is obtained as a Kronecker product of the irreducible real representations of $\mathbf{P}, \mathbf{S}$, and $\mathbf{T} ; \check{G}=\check{P} \otimes \check{S} \otimes \check{T}$. $\check{P}$ is characterized by an ordering vector $q$ in the Brillouin zone and an irreducible representation of its little group $\mathbf{P}(q)$, and is therefore labeled $q \check{P}(q)$. We denote the relevant representations of $\mathbf{S}$ by $\check{S}^{0}$ (singlet) and $\breve{S}^{1}$ (triplet), while those of $\mathbf{T}$ by $\check{T}^{0}$ (symmetric) and $\check{T}^{1}$ (antisymmetric). $\check{P} \otimes \check{S}^{0} \otimes \check{T}^{0}$, $\check{P} \otimes \check{S}^{1} \otimes \check{T}^{1}, \check{P} \otimes \check{S}^{0} \otimes \check{T}^{1}$, and $\check{P} \otimes \check{S}^{1} \otimes \check{T}^{0}$, respectively, describe charge-density-wave (CDW), spin-density-wave (SDW), charge-current-wave (CCW), and spin-currentwave (SCW) states. We leave out all the current-wave states, which either result in the one-way uniform flow or break the charge-conservation law in the present system. We consider density waves of two types, $q=0$ and $q=\pi$, which are labeled $\Gamma$ and $X$, respectively. Thus the representations labeled $K \check{P}(K) \otimes \check{S}^{i} \otimes \check{T}^{i}(K=\Gamma, X$; $i=0,1)$ are of our interest. Since $\mathbf{P}(\Gamma)=\mathbf{P}(X)=\mathbf{C}_{2}$, $\check{P}(\Gamma)$ and $\check{P}(X)$ are either $A\left(C_{2^{-}}\right.$symmetric $)$or $B\left(C_{2^{-}}\right.$ antisymmetric) representation.

Then the Hartree-Fock (HF) approximation of the Hamiltonian (11) in the momentum space is given by

$\mathcal{H}_{\mathrm{HF}}=\sum_{K=\Gamma, X} \sum_{\lambda=0, z} \sum_{i, j} \sum_{k, s, s^{\prime}} x_{i j}^{\lambda}(K ; k) a_{i: k+q, s}^{\dagger} a_{j: k, s^{\prime}} \sigma_{s s^{\prime}}^{\lambda}$.

$x_{i j}^{\lambda}(K ; k)$ is self-consistently given in terms of the density matrices $\rho_{i j}^{\lambda}(K ; k)=\frac{1}{2} \sum_{s, s^{\prime}}\left\langle a_{j: k+q, s}^{\dagger} a_{i: k, s^{\prime}}\right\rangle_{\mathrm{HF}} \sigma_{s s^{\prime}}^{\lambda}$, where the $2 \times 2$ matrices $\sigma^{\lambda}$ are the unit $(\lambda=0)$ and Pauli $(\lambda=x, y, z)$ matrices, and $\langle\cdots\rangle_{\mathrm{HF}}$ means the quantum average in a HF eigenstate. We note that no helical-spin $(\lambda=x, y)$ solution is obtained from the present model. When $\mathcal{H}_{\mathrm{HF}}$ is decomposed into spatial-symmetry-definite components as

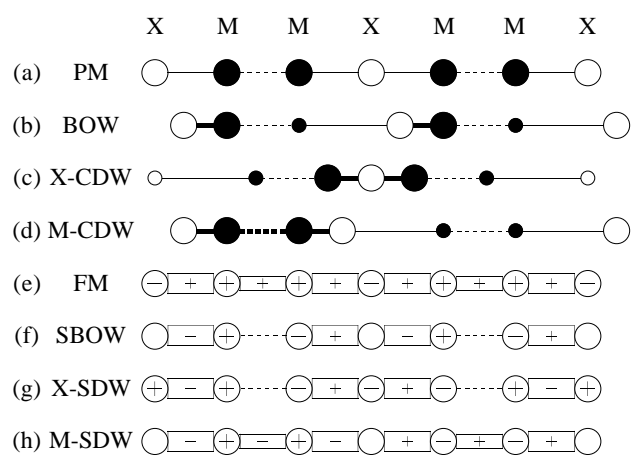

FIG. 1. Schematic representation of possible density-wave states, where the variety of circles and segments qualitatively represents the variation of local charge densities and bond orders, respectively, whereas the signs \pm in circles and strips describe the alternation of local spin densities and spin bond orders, respectively. Circles shifted from the regular position qualitatively represent lattice distortion, which is peculiar to nonmagnetic phases.

$$
\mathcal{H}_{\mathrm{HF}}=\sum_{D=A, B} \sum_{K=\Gamma, X} \sum_{\lambda=0, z} h^{\lambda}(K ; D)
$$

the broken-symmetry Hamiltonian for the representation $K D \otimes \check{S}^{i} \otimes \check{T}^{i}$ is given by $h^{0}(\Gamma ; A)+h^{\lambda}(K ; D)$, where $\lambda=0$ for $i=0$ and $\lambda=z$ for $i=1$. The charge $(\lambda=0)$ and spin $(\lambda=z)$ densities on site $i$ in the $n$th MXM unit are expressed as $d_{i: n}^{\lambda}=\frac{1}{2} \sum_{s, s^{\prime}}\left\langle a_{i: n, s}^{\dagger} a_{i: n, s^{\prime}}\right\rangle_{\mathrm{HF}} \sigma_{s s^{\prime}}^{\lambda}$, while the bond $(\lambda=0)$ and spin bond $(\lambda=z)$ orders between site $i$ in the $n$th MXM unit and site $j$ in the $m$ th MXM unit are defined as $p_{i: n ; j: m}^{\lambda}=\frac{1}{2} \sum_{s, s^{\prime}}\left\langle a_{i: n, s}^{\dagger} a_{j: n, s^{\prime}}\right\rangle_{\mathrm{HF}} \sigma_{s s^{\prime}}^{\lambda}$. The lattice displacements $u_{i: n}$ are self-consistently determined so as to minimize the HF energy $E_{\mathrm{HF}} \equiv\langle\mathcal{H}\rangle_{\mathrm{HF}}$. Further group-theoretical procedure will be detailed elsewhere. Here we briefly characterize all the phases and schematically show them in Fig. 1.

(a) $\Gamma A \otimes \check{S}^{0} \otimes \check{T}^{0}$ : The paramagnetic state with the full symmetry $\mathbf{G}$, abbreviated as PM.

(b) $\Gamma B \otimes \check{S}^{0} \otimes \check{T}^{0}$ : Bond order wave with polarized charge densities in the M sublattice, abbreviated as BOW.

(c) $X A \otimes \check{S}^{0} \otimes \check{T}^{0}$ : Charge density wave on the $\mathrm{X}$ sublattice with alternating polarized charge densities in the $\mathrm{M}$ sublattice, abbreviated as X-CDW.

(d) $X B \otimes \check{S}^{0} \otimes \check{T}^{0}$ : Charge density wave on the M sublattice, abbreviated as M-CDW.

(e) $\Gamma A \otimes \check{S}^{1} \otimes \check{T}^{1}:$ Ferromagnetism with uniform spin bond orders, abbreviated as FM.

(f) $\Gamma B \otimes \check{S}^{1} \otimes \check{T}^{1}$ : Spin bond order wave with polarized spin densities in the M sublattice, abbreviated as SBOW.

(g) $X A \otimes \check{S}^{1} \otimes \check{T}^{1}$ : Spin density wave on the X sublattice with alternating polarized spin densities in the M sublattice, abbreviated as X-SDW.

(h) $X B \otimes \check{S}^{1} \otimes \check{T}^{1}$ : Spin density wave on the M sublattice, abbreviated as M-SDW.

The nonmagnetic phases (a)-(d), respectively, correspond to the averaged-valence, charge-polarization, alternating charge-polarization, and CDW states in the experimental convention 111, the latter three of which exhibit el-ph coupling. $\mathrm{X}-\mathrm{CDW}$ is a novel density-wave state stabilized by M-sublattice distortion. It is the case for the MX-type complexes that the metal ions are locked in the surrounding ligands. Although the binuclear metal units are still heavily surrounded by ligands, ${ }^{129} \mathrm{I}$ Mössbauer spectroscopy measurements 111] for $\mathrm{Pt}_{2}(\mathrm{dta})_{4} \mathrm{I}$ imply the ground state of the X-CDW type. M-sublattice distortion has not yet been reported for the pop series, where the ground state of the M-CDW type appears [10]. This competition is a central issue in our argument. On the other hand, the CDW-SDW competition is another interesting issue. Magnetic instabilities are generally not coupled with phonons. 


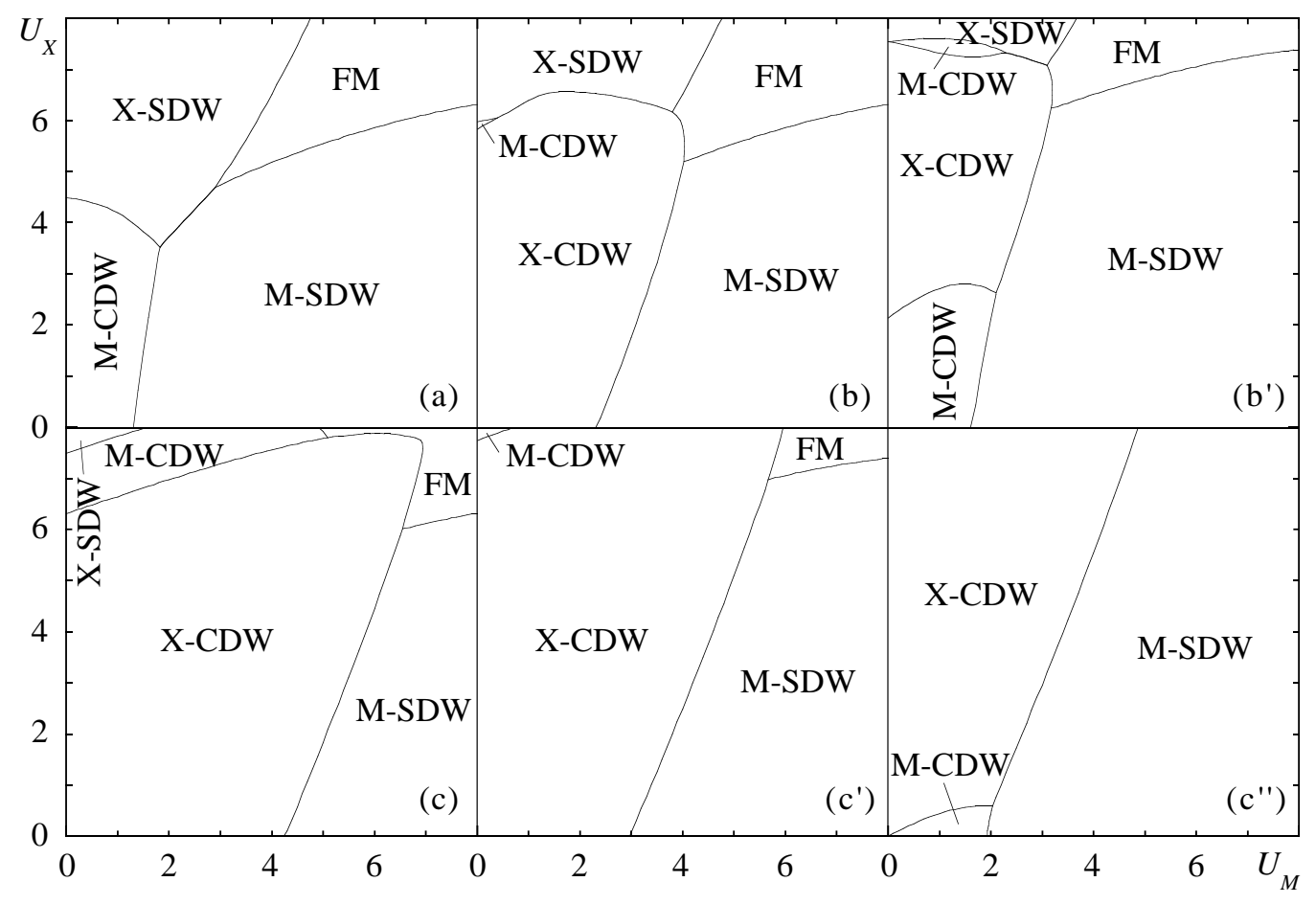

FIG. 2. Typical ground-state phase diagrams at $\frac{5}{6}$ band filling, where the parametrization, $V_{\mathrm{MM}}=V_{\mathrm{Mx}}=1.0$, is common to all. (a) $\alpha=0.9, \beta=0.6, \varepsilon_{\mathrm{M}}-\varepsilon_{\mathrm{X}}=1.0$. (b) $\alpha=1.2, \beta=0.6, \varepsilon_{\mathrm{M}}-\varepsilon_{\mathrm{X}}=1.0 ;\left(\mathrm{b}^{\prime}\right) \alpha=1.2, \beta=0.6, \varepsilon_{\mathrm{M}}-\varepsilon_{\mathrm{X}}=2.0$. (c) $\alpha=1.5, \beta=0.6, \varepsilon_{\mathrm{M}}-\varepsilon_{\mathrm{X}}=1.0 ;\left(\mathrm{c}^{\prime}\right) \alpha=1.5, \beta=0.6, \varepsilon_{\mathrm{M}}-\varepsilon_{\mathrm{X}}=2.0 ;\left(\mathrm{c}^{\prime \prime}\right) \alpha=1.5, \beta=0.6, \varepsilon_{\mathrm{M}}-\varepsilon_{\mathrm{X}}=3.0$.

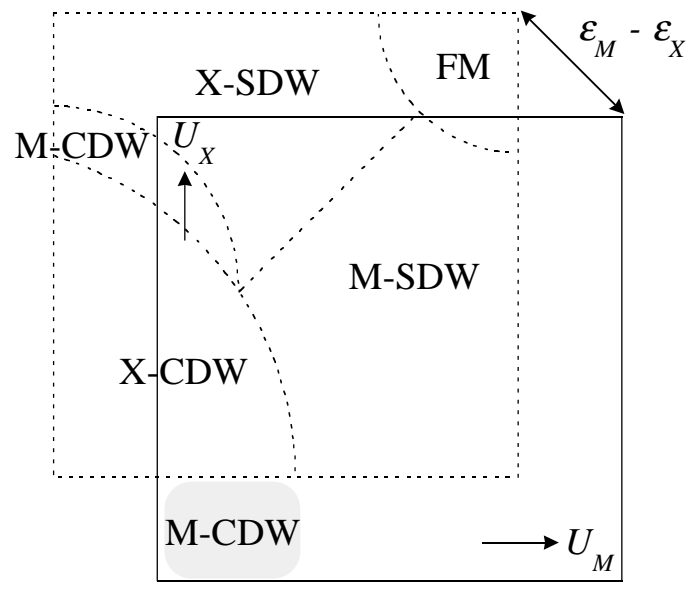

FIG. 3. Qualitative understanding of the phase diagrams at $\frac{5}{6}$ band filling.

Let us proceed to numerical investigations. We draw ground-state phase diagrams by calculating $E_{\mathrm{HF}}$ in the thermodynamic limit. We show in Fig. 2 typical phase diagrams at $\frac{5}{6}$ band filling, which describe the as-grown MMX compounds. As for CDW states, M-CDW is stabilized in the small- $\alpha$ region, while it is mostly replaced by X-CDW with the increase of $\alpha$. When the $\mathrm{X} p p_{z}$ orbital stays far bellow the $\mathrm{M} d_{z^{2}}$ orbital, a certain Coulomb repulsion on the $\mathrm{X}$ site is necessary for the stabilization of
$\mathrm{X}-\mathrm{CDW}$, which is accompanied by the alternating charge densities on the $\mathrm{X}$ sublattice and therefore needs the driving force $U_{\mathrm{X}}$ to push up the $\mathrm{X} p_{z}$ electrons. On the other hand, the SDW states are stabilized for large enough $U_{\mathrm{M}}$ or $U_{\mathrm{X}}$. Ni complexes, whose $U_{\mathrm{M}}$ is relatively large, may be candidates for M-SDW. Lower-lying $\mathrm{X} p_{z}$ orbitals less stabilize X-SDW whose $\mathrm{X} p_{z}$ orbitals are not fully occupied. Fluctuations of the X-SDW type are interesting when $\varepsilon_{\mathrm{M}}$ and $\varepsilon_{\mathrm{X}}$ are close enough. The present observations are qualitatively summarized in Fig. 3. $U_{\mathrm{M}}$ favors M-SDW, while $U_{\mathrm{X}}$ is advantageous to X-SDW. The CDW states both dislike the on-site Coulomb repulsions, especially $U_{\mathrm{M}}$. M-CDW and X-CDW are almost equal with respect to $U_{\mathrm{M}}, U_{\mathrm{X}}$, and $V_{\mathrm{MX}}$, but they are distinguished by $V_{\mathrm{MM}}$. Therefore, unless $\alpha$ is small enough, $\mathrm{X}-\mathrm{CDW}$ is more stabilized. With the increase of $\varepsilon_{M}-\varepsilon_{X}$, the density waves on the $\mathrm{X}$ sublattice are generally reduced. Within the mean-field approximation, the Stoner's ferromagnetism is necessarily stabilized for large enough $U_{\mathrm{M}}$ and $U_{\mathrm{X}}$.

Finally, we mention the hole-doped system. Typical phase diagrams at $\frac{4}{6}$ and $\frac{3}{6}$ band fillings are shown in Fig. 4. Considering the nesting vector for the Fermi surfaces, it is convincing that density waves of $q=\pi$ appear at $\frac{5}{6}$ and $\frac{3}{6}$ band fillings, while they are less stabilized at $\frac{4}{6}$ band filling. In this context, let us consider the electronic structures, which are illustrated in Fig. 5 at the idea of the same-valence metals forming mixed orbitals [11]. The point is that the highest $d_{\sigma^{*}}$ orbitals are all 
empty in any $q=\pi$ density-wave state, while they are occupied by finite electrons in any $q=0$ density-wave state. Under the doping of a hole per unit cell, $q=0$ states can closely compete with $q=\pi$ states.

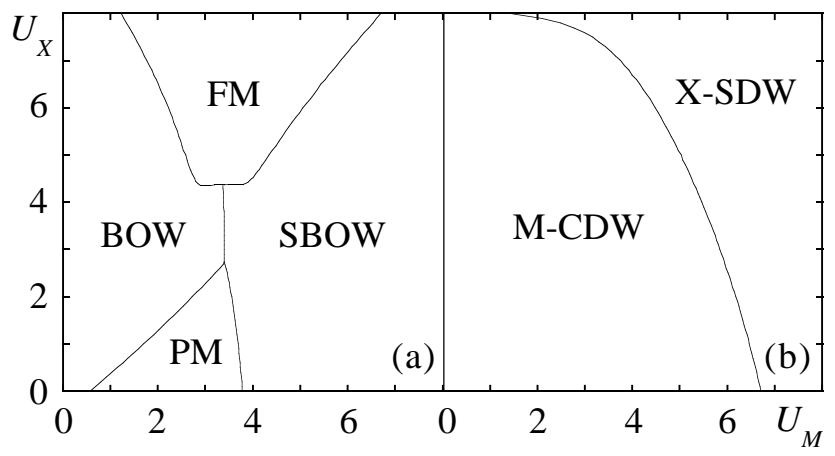

FIG. 4. Typical ground-state phase diagrams under hole doping, where $V_{\mathrm{MM}}=V_{\mathrm{MX}}=1.0, \alpha=0.9, \beta=0.6$, and $\varepsilon_{\mathrm{M}}-\varepsilon_{\mathrm{X}}=1.0$. (a) $\frac{4}{6}$ band filling. (b) $\frac{3}{6}$ band filling.

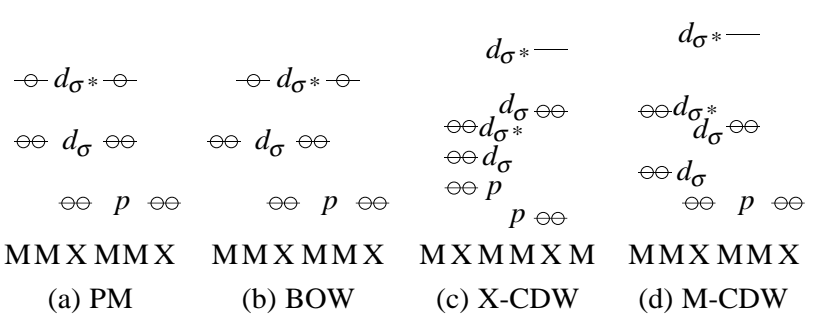

FIG. 5. Schematic representation of the electronic structures of the MMX chain in the ground state of the nonmagnetic density-wave type in the case of neglecting any Coulomb correlation.

Physical research on MMX compounds seems to be still in its early stage. The likely ground state of the X-CDW type in $\mathrm{Pt}_{2}(\mathrm{dta})_{4} \mathrm{I}$ has quite recently been supported by a single-band-model calculation 18 as well. However, from the experimental point of view, further verification, such as Raman spectroscopy and X-ray structural analysis, may be provided. The mobile bimetallic units are still wrapped in mystery. We hope the present calculations will motivate and accelerate further synthesis and measurements of these fascinating materials.

It is a pleasure to thank Prof. H. Okamoto, Prof. K. Yonemitsu, and Dr. M. Kuwabara for fruitful discussions. This work was supported by the Japanese Ministry of Education, Science, and Culture, and the SanyoBroadcasting Foundation for Science and Culture. The numerical calculation was done using the facility of the
Supercomputer Center, Institute for Solid State Physics, University of Tokyo.

[1] R. J. H. Clark, in Advances in Infrared and Raman Spectroscopy, edited by R. J. H. Clark and R. E. Hester (Wiley, New York, 1984) Vol. II, p. 95.

[2] H. Toftlund and O. Simonsen, Inorg. Chem. 23 (1984) 4261.

[3] K. Toriumi, Y. Wada, T. Mitani, S. Bandow, M. Yamashita, and Y. Fujii, J. Am. Chem. Soc. 111 (1989) 2341.

[4] H. Okamoto, T. Mitani, K. Toriumi, and M. Yamashita, Phys. Rev. B 42 (1990) 10381.

[5] K. Nasu, J. Phys. Soc. Jpn. 52 (1983) 3865.

[6] J. T. Gammel, A. Saxena, I. Batistić, A. R. Bishop, and S. R. Phillpot, Phys. Rev. B 45 (1992) 6408.

[7] I. Batistić, J. T. Gammel, and A. R. Bishop, Phys. Rev. B 44 (1991) 13228.

[8] H. Röder, A. R. Bishop, and J. T. Gammel, Phys. Rev. Lett. 70 (1993) 3498.

[9] S. D. Conradson, M. A. Stroud, M. H. Zietlow, B. I. Swanson, D. Baeriswyl, and A. R. Bishop, Solid State Commun. 65 (1988) 723; B. I. Swanson, M. A. Stroud, S. D. Conradson, and M. H. Zietlow, ibid. 65 (1988) 1405.

[10] N. Kimura, H. Ohki, R. Ikeda, M. Yamashita, Chem. Phys. Lett. 220 (1994) 40.

[11] H. Kitagawa, N. Onodera, T. Sonoyama, M. Yamamoto, M. Fukawa, T. Mitani, M. Seto, and Y. Maeda, preprint, to be published in J. Am. Chem. Soc..

[12] C.-M. Che, F. H. Herbstein, W. P. Schaefer, R. E. Marsh, and H. B. Gray, J. Am. Chem. Soc. 105 (1983) 4604; L. G. Butler, M. H. Zietlow, C.-M. Che, W. P. Schaefer, S. Sridhar, P. J. Grunthaner, B. I. Swanson, R. J. H. Clark, and H. B. Gray, ibid. 110 (1988) 1155.

[13] M. Kurmoo and R. J. H. Clark, Inorg. Chem. 24 (1985) 4420; R. J. H. Clark, M. Kurmoo, H. M. Dawes, and M. B. Hursthouse, ibid. 25 (1986) 409.

[14] C. Bellitto, A. Flamini, L. Gastaldi, and L. Scaramuzza, Inorg. Chem. 22 (1983) 444; C. Bellitto, G. Dessy, and V. Fares, ibid. 24 (1985) 2815.

[15] M. Ozaki, Int. J. Quantum Chem. 42 (1992) 55; S. Yamamoto and M. Ozaki, ibid. 44 (1992) 949.

[16] S. Yamamoto and M. Ozaki, Solid State Commun. 83, 329 (1992) 329; 335.

[17] S. Yamamoto, Phys. Lett. A 247 (1998) 422; 258 (1999) 183.

[18] M. Kuwabara and K. Yonemitsu, preprint, to be published in Mol. Cryst. Liq. Cryst.. 